FEDSM2005-77015

\title{
NUMERICAL ANALYSIS OF FLOW PHENOMENA RELATED TO THE UNSTABLE ENERGY-DISCHARGE CHARACTERISTIC OF A PUMP-TURBINE IN PUMP MODE
}

\author{
O. Braun J.-L. Kueny F. Avellan \\ École Polytechnique Fédérale de Lausanne \\ Laboratoire de Machines Hydrauliques - STI-LMH \\ $\mathrm{CH}-1015$ Lausanne, Switzerland
}

\section{ABSTRACT}

Regions of positive slope in the pressure-discharge characteristics are one of the major concerns in design and operation of centrifugal pumps, as they are limiting the admissible operating range to values above the critical discharge.

The industrial pump turbine of specific speed $v=0.42\left(n_{q}=66\right.$ $\mathrm{min}^{-1}$ ) proposed as QNET-CFD test case TA6-04 shows a marked saddle in the energy-discharge characteristic associated to a sudden drop of efficiency versus discharge at part load. The pump-turbine consists of a shrouded impeller with five blades, a diffuser with 22 guide and stay vanes and a spiral casing.

CFD flow simulations on a reduced model were carried out with a finite volume Navier-Stokes code (CFX-5.7) using block-structured hexahedral meshes and the Menter-SST Turbulence model. Control of numerical quality has been performed.

Reduced models with relatively low computational effort (mixing plane interface) already permit to capture the drop in efficiency and energy coefficient to analyze the flow phenomena inducing the drop of the energy coefficient $\Psi$ that occurs at partial discharge.

Analysis of local flow patterns and energy and velocity distributions at the rotor-stator interface that are related to the onset of recirculation are presented.

\section{INTRODUCTION}

The investigated industrial pump-turbine of specific speed $v=0.42\left(n_{q}=66 \min ^{-1}\right)$ has been investigated experimentally by means of global performance measurements and local LDV measurements at guide vane inlet in pump mode and 2D-PIV measurements in the guide vane channels. They exposed the onset of a recirculating area at the hub side of the guide vanes that is related to the drop in the energy-discharge and the efficiency-discharge characteristics [1].
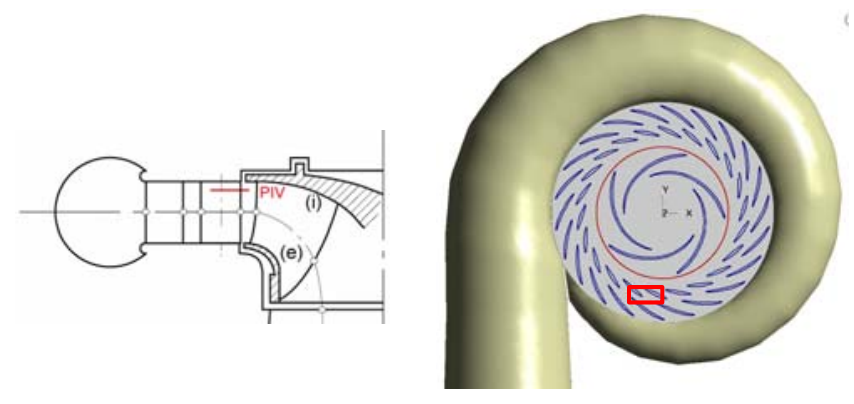

Fig. 1. Location of the PIV measurement zone
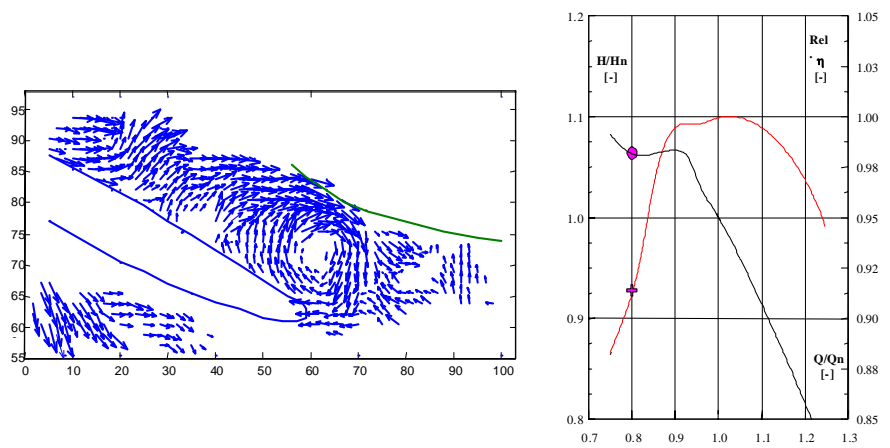

Fig. 2. Onset of recirculation at the guide vane hub side related to the drop in the characteristic

Numerical studies were carried out on the pump-turbine geometry within the scope of development of unsteady rotor stator techniques [2], but without addressing in details the alteration of the characteristic curve in part load regime and the associated flow patterns. Within the framework of a project assessing the use of CFD simulations for part load operations of centrifugal pumps, the presented pump turbine constitutes the main test case. 


\section{NOMENCLATURE}

C velocity

$\mathrm{C}_{\mathrm{p}} \quad$ dimensionless pressure

$\mathrm{c}_{\mathrm{r}} \quad$ radial velocity

$\mathrm{c}_{\mathrm{u}} \quad$ circumferential velocity

$\mathrm{n}_{\mathrm{q}} \quad$ specific speed $\left(\mathrm{min}^{-1}\right)$

$\mathrm{p} \quad$ static pressure

Q volume flow rate

$\mathrm{Q}_{\mathrm{c}}$ channel flow rate

$Q_{p} \quad$ volume flow rate through periodic interface

$\mathrm{Q}_{\mathrm{p}}{ }^{*} \quad$ volume flow ratio interface - channel

$\mathrm{R}$ radius

$\mathrm{R}_{1 . \mathrm{e}} \quad$ outer impeller diameter at shroud

$\mathrm{y}^{+}$dimensionless wall distance

$v \quad$ specific speed

$\rho$ density

$\varphi$ discharge coefficient

$\psi \quad$ energy coefficient (global)

$\psi_{1} \quad$ rotor outlet energy coefficient

$\psi_{\mathrm{k}}{ }^{*} \quad$ local relative kinetic energy coefficient

$\psi_{\mathrm{p}}^{*} \quad$ local relative pressure energy coefficient

$\omega \quad$ vorticity

$\omega_{\mathrm{s}} \quad$ streamwise vorticity

$\omega_{\mathrm{s}, \mathrm{rel}}$ relative streamwise vorticity

\section{NUMERICAL METHOD}

As a first step in the investigation of the capabilities of numerical methods in part load pump operation, steady state simulations with a reduced domain were carried out, using only one channel per component with a circumferentially averaged interface between the impeller and the stator, which is appropriate for applications with a relatively large mixing distance between the rotating and the stationary blade edges [3]. The spiral casing is not modeled and replaced by a converging revolution body as an outlet region in order to achieve numerical stability even for part load operating points. The meridional view of the domain (figure 3) shows the location of boundary conditions and of the rotor-stator interfaces between the three modeled domains.

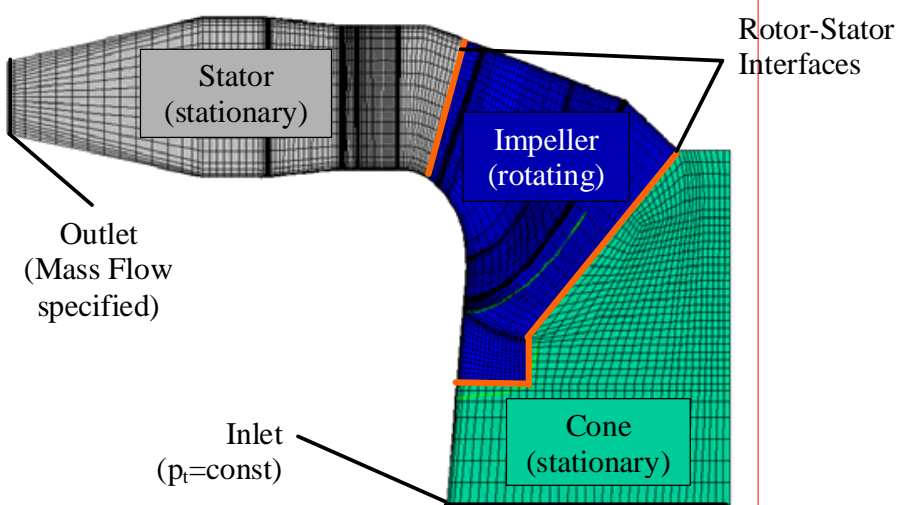

Fig. 3. Domains, interfaces and boundary conditions
Amongst the available two-equation RANS turbulence models the SST-Model is chosen. It is using a $\mathrm{k}-\varepsilon$ formulation in the free shear flow and a $\omega$-based formulation in the nearwall region. The wall treatment switches between a log-law based formulation if the nearest node at the wall is at $\mathrm{y}^{+}>11$ and a consistent $\omega$-based integration up to the wall node for $\mathrm{y}^{+}<=11$ [4].

The hexahedral meshes were generated using a generic blocktopology based meshing tool (ICEM Hexa) and a family of topology templates developed for turbomachine blade passages. The topologies use an O-type grid around the blades combined with quarter-o-grids to match the geometric constraints while guaranteeing high element skew angles.

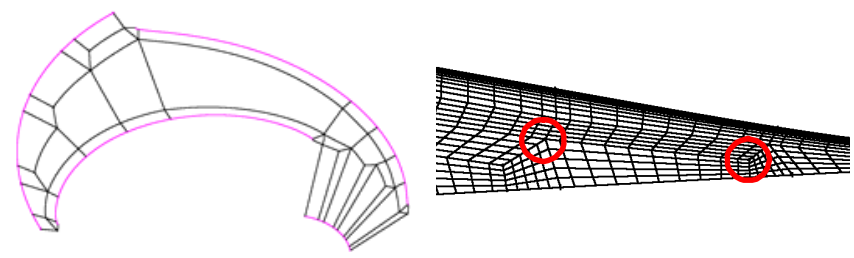

\section{Fig. 4: Block structured topology and volume size ratios at topology vertices}

The lowest angles for the rotor blade channel mesh are over $30^{\circ}$ and all volume elements in the surface boundary blocks have skew angles over $60^{\circ}$. Particular care was taken to realize smooth changes in volume edge length at the topology vertices.

By consistent scaling of basic block element numbers, meshes of three different refinement degrees were generated, leading to average $\mathrm{y}^{+}$values on the blade surfaces of 100, 20 and 5 for the coarse, medium and fine grids.

\begin{tabular}{|l|l|l|l|}
\hline & Impeller & Stator & Cone \\
\hline Coarse & 160.000 & 55.000 & 30.000 \\
\hline Medium & 455.000 & 180.000 & 100.000 \\
\hline Fine & 1.550 .000 & 550.000 & 300.000 \\
\hline
\end{tabular}

Table 1: Mesh sizes of the three components for different refinement degrees

Simulations for different operating points were carried out using the three different mesh sizes. The advection scheme is based on an upwind scheme adding correction terms for the control volume interface fluxes based on trilinear interpolation. Using a so called blend factor of 1.0 as a multiplier for the correction terms, which is formally second order accurate, leads to insufficient convergence behavior in some operating points. Only $90 \%$ (Blend Factor of 0.9 ) of these correction terms were actually added, convergence in 100 to 150 timesteps was achieved.

Iteration error was checked and a maximum normalized residual of $5.10^{-4}$ was fixed as a sufficient criterion based on the global energy coefficient variation being less then $0.1 \%$. The global energy coefficient and efficiency were monitored during each run. For some well defined discharge values 
$(\varphi=0.10)$, where the drop in the characteristic curves occurs, there was no convergence towards a steady solution, but the global performance values were oscillating between the values found for the neighboring operating points. There was no reproducible hysteresis detected when starting the same simulations with initial conditions from lower respectively higher discharge values.

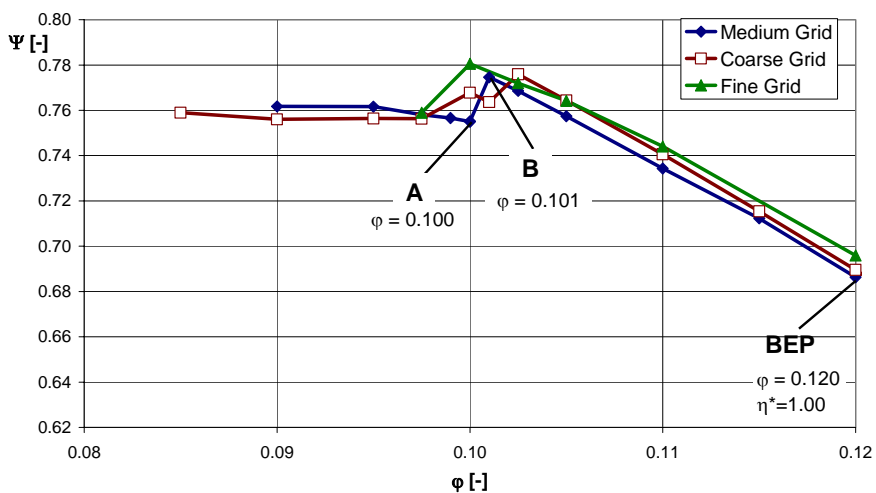

Fig. 5. Grid convergence study based on global energy coefficient

The grid convergence study shows that the qualitative behavior or the drop in the energy-discharge characteristic is reproduced with different spatial discretisation refinement at similar discharge values. The important difference of this discharge value when compared to experimental characteristic measurement (where the drop occurs at $\varphi=0.11$ ) is not reduced by using finer grids. The further analysis is achieved with the operating points $\mathrm{A}(\varphi=0.100)$ and $\mathrm{B}(\varphi=0.101)$ computed on the medium grids.

\section{ANALYSIS OF FLOW PATTERNS}

For the analysis of three-dimensional detached flow, skin friction lines that are analogous to experimental oil film patterns allow a good insight and comprehension of the global flow topology [5]. This technique has been successfully used to visualize the appearance of a three-dimensional separation of Werlé-Legendre type related to a drop in the global performance of a Francis turbine draft tube [6]. Surface friction lines are represented for different discharge values on the guide and stay vane suction side on the modeled stator channel.
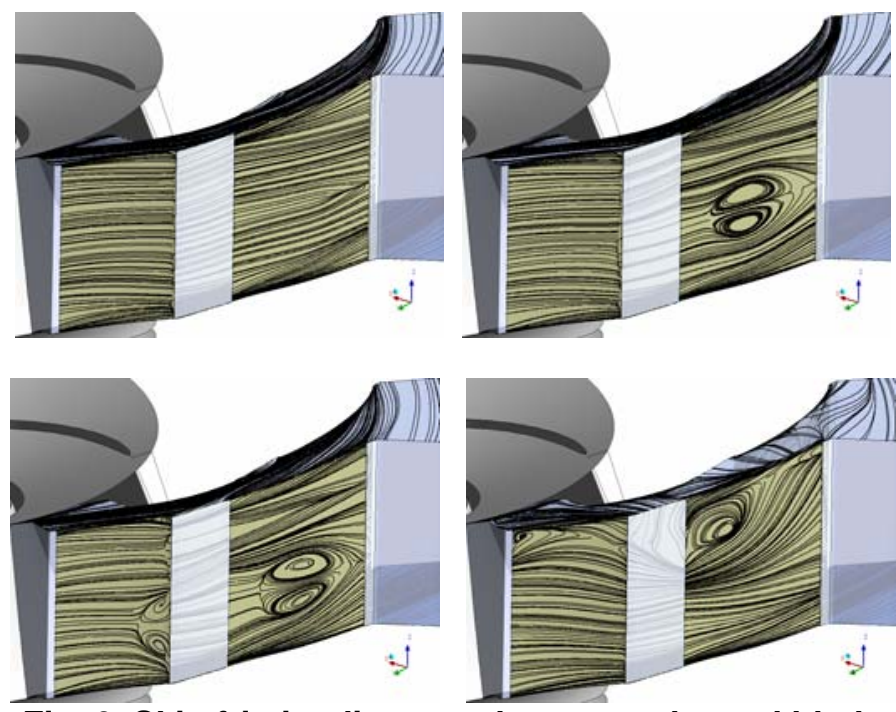

Fig. 6. Skin friction lines on the stator channel blade and hub surfaces $a) \varphi=0.115 \mathrm{~b}) \varphi=0.110 \mathrm{c}) \varphi=0.101 \mathrm{~d}$ ) $\varphi=0.100$

At the first operating point computed below the best efficiency point $(\varphi=0.115)$, the skin friction lines on the guide vane suction side converge towards a line, which indicates the beginning of a flow separation in the middle of the channel.

This region of detached flow develops towards lower discharge value $(\varphi=0.110)$, and the detachment is appearing on the guide vane suction side also at $\varphi=0.101$.

The appearance and growth of these detached flow zones has no impact on the computed global performances. The separation regions are closed volumes attached to a blade surface and do not exchange and dissipate significantly more energy from the sound through-flow than a solid wall does.

Closely above the critical discharge value $(\varphi=0.101)$, a second separation line near the hub is found on the stay vane suction side appears.

At the critical discharge value $(\varphi=0.100)$ the central separation region disappears giving place to an extended recirculation zone at the stator hub side. The skin friction line pattern on the stator hub surface indicates completely reversed flow pattern in this region, as it is not oriented with the main flow direction. This bifurcation of the flow topology results in a sudden drop of the energy coefficient, because the flow structure that appears extends through the entire channel from the guide vane leading edge to the outlet.

\section{ANALYSIS OF ENERGY TERMS ALONG THE STATOR CHANNEL}

In the present case, a recirculating vortex zone characterized by low hydraulic energy appears, blocking a part of the diffuser channels. Figure 6 shows a comparison of the mass-flow-averaged energy quantities in the diffuser for the two operating points. 


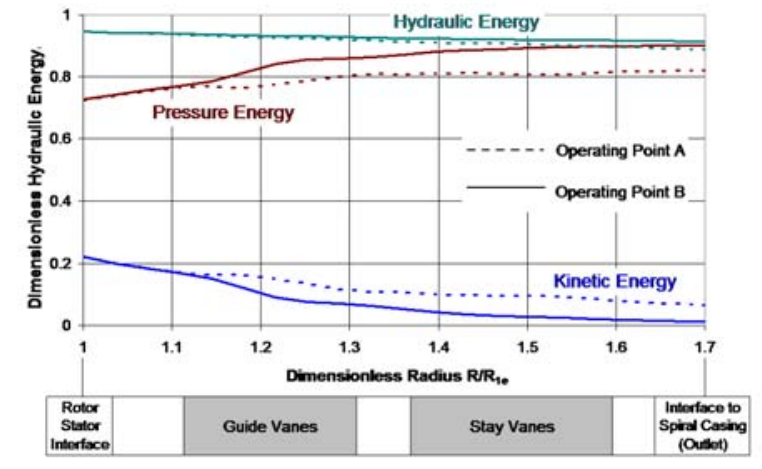

Fig. 7. Streamwise distribution of hydraulic, kinetic and pressure energy in the stator

For operating point $A$, the recovery of kinetic energy to pressure energy in the guide vane zone is considerably lower than for point $\mathrm{B}$ and the leftover kinetic energy cannot be recovered to pressure energy further downstream in the diffuser.

One part of this extra kinetic energy is lost in the remaining diffuser by dissipation in the secondary flow structures.

Another considerable part of this kinetic energy is still available as such at the high pressure stay vane section. It cannot be presumed that this part of kinetic energy will be recovered in the spiral casing, so the drop in energy coefficient and efficiency is possibly higher when considering the entire machine.

\section{IMPELLER OUTLET ENERGY PROFILES}

The development of secondary flow in the stator channel is mainly influenced by the upstream velocity and pressure distribution. Following [6], the development of secondary flows in a curved channel is increased by the non-uniformity of the specific hydraulic energy in the inlet section.

To analyze the non-uniformity of the energy distribution on the circumferentially averaged rotor-stator interface, we consider the local energy coefficient $\psi_{\text {local }}$ divided by the rotor outlet energy coefficient $\psi_{1}$ obtained by mass-flow weighted average on the interface surface.

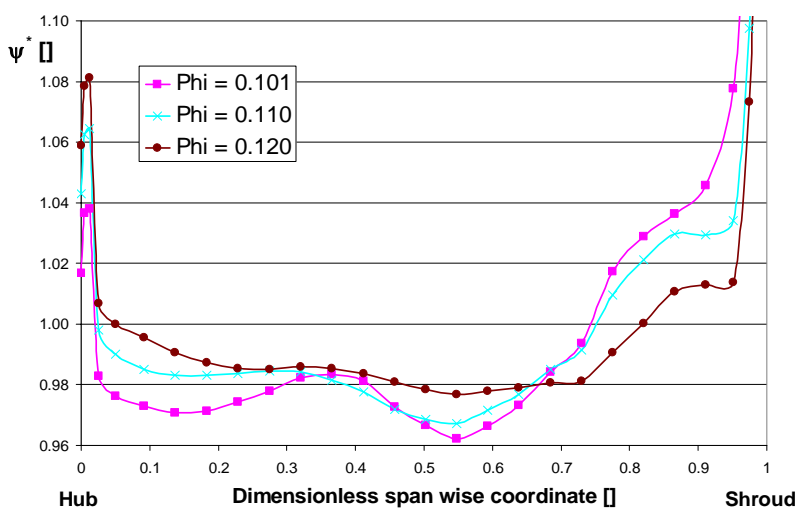

Fig. 8. Hydraulic energy profiles at impeller outlet

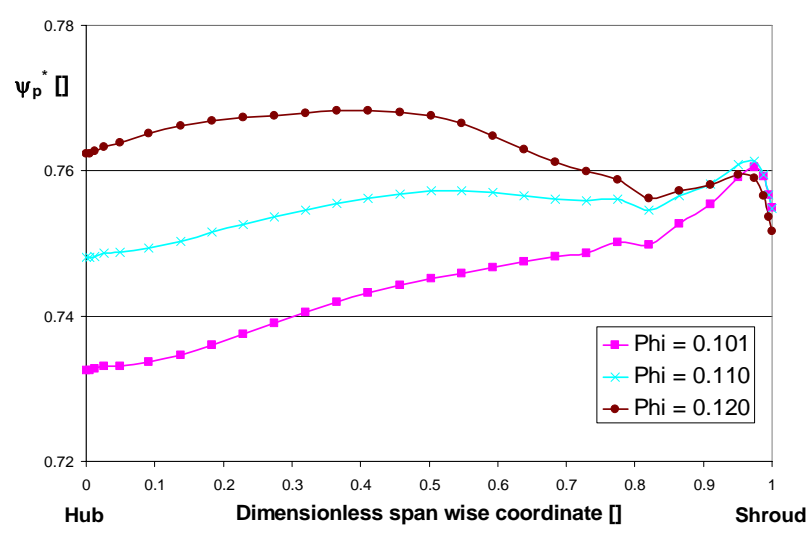

Fig. 9. Pressure energy profiles at impeller outlet

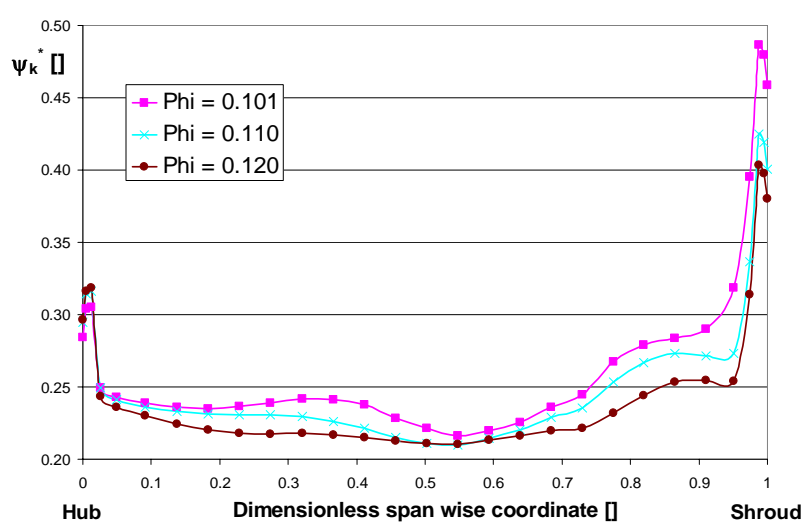

Fig. 10. Kinetic energy profiles at impeller outlet

Towards lower discharge value, the hydraulic energy distribution at impeller outlet becomes more and more asymmetric, the hydraulic energy near the shroud increases towards lower discharge whereas it decreases at the hub. However, the absolute minimum of hydraulic energy is located in the middle of the channel at all discharge values.

The continuous rising of the span wise disequilibrium of hydraulic energy at impeller outlet can serve as a first explanation of the change in flow patterns observed. A decomposition of the local energy coefficient $\psi_{\text {local }}$ into the pressure part $\psi_{\text {p.local }}$ and the kinetic energy part $\psi_{\text {k.local }}$ shows that the relative contribution of pressure energy to the total energy lowers at the hub towards lower discharge, by consequence, the adverse pressure gradient in the hub boundary layer is increasing, giving rise to the massive flow separation occurring at operating point A.

\section{IMPELLER OUTLET VELOCITY PROFILES}

The velocity profiles at impeller outlet are characterized by a low radial velocity component near the shroud. Accordingly, the circumferential component is higher then the average. 


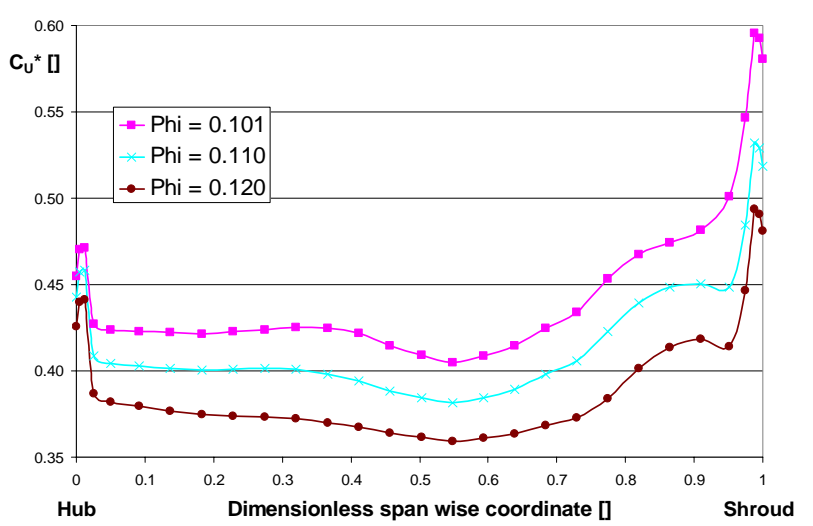

Fig. 11. Circumferential velocity profiles at impeller outlet

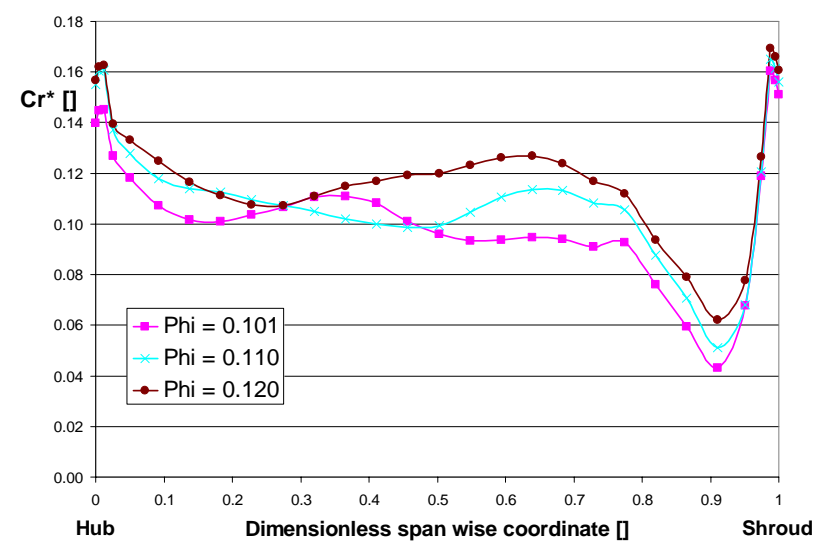

Fig. 12. Radial velocity profiles at impeller outlet

This could lead at a first glance in an incidence error on the guide vane trailing edge, but this does not result in a flow detachment near the shroud. The radial equilibrium of the flow is given by:

$$
\left(\frac{\partial p}{\partial r}\right)=\frac{\rho c_{u}{ }^{2}}{R}
$$

Due to the repartition of the static pressure at the rotor outlet, the radial pressure gradient between rotor outlet and the guide vane channel entry is lower in the shroud then in the hub section, furthermore, the circumferential velocity is higher. So the apparent incidence error does not cause flow detachment on the guide vane suction side, as the flow does locally not follow a logarithmic spiral, but the flow angle is increasing towards higher radii as the streamlines approach straight lines. For reasons of continuity, the convergence of the meridional flow due to this acceleration of the meridional component needs to be compensated by the surrounding flow. The skin friction lines in Fig. 6 b) and c) show that effect in the lower part of the channel.

\section{SECONDARY FLOW PATTERNS}

The guide and stay vane configuration does not form a closed channel but allows the fluid to cross from one channel to another depending on the local momentum equilibrium. This makes a comparison of the secondary flow patterns difficult as the flow is redefining a different channel when changing configuration. This is illustrated by the change in the volume flow rate $\mathrm{Q}_{\mathrm{p}}$ through the periodic interface corresponding to the passage between the guide and the stay vane made dimensionless by the channel flow rate $\mathrm{Q}_{c}$ :
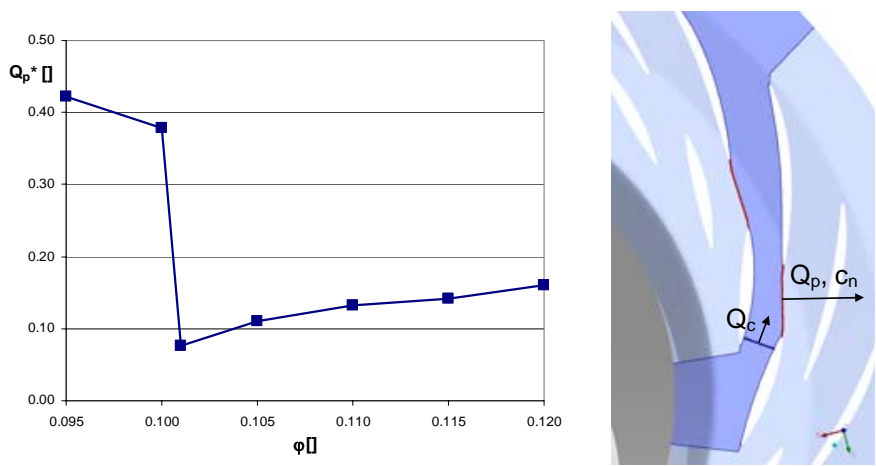

Fig. 13.: Relative flow rate through the periodic interface between guide and stay vanes

In the operating points above the drop in the characteristic curves, only a relatively small part of the flow rate is transiting from one channel to another. This part is decreasing towards lower discharge, which means that the average flow angle in these regions is decreasing too. The flow traversing the interface is governed by the equilibrium between the pressure gradient between the two channels, the upstream velocity and the streamline curvature.

The pressure gradient in the passage between two channels is due to the staggered configuration together with the pressure rise in the channel. As long as the transformation of kinetic energy to pressure energy by diffusion in the channel takes place without excessive separation, there is a pressure gradient that contributes to avoid the flow from crossing the interface.
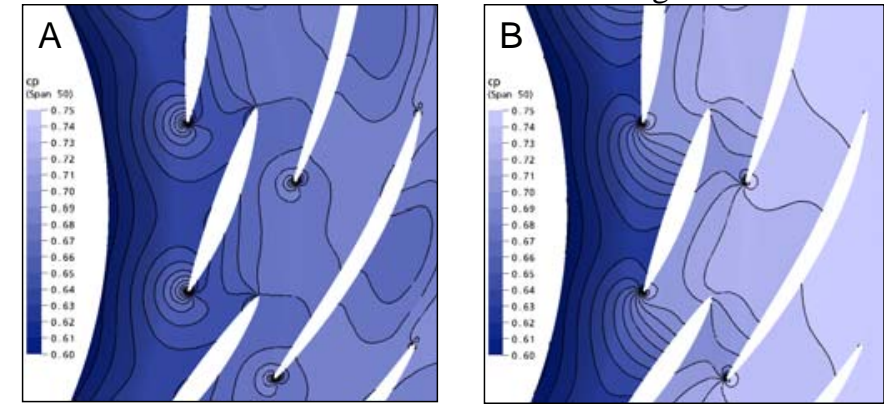

Fig. 14. Pressure contours in the mid-span blade-toblade plane for operating points $A$ and $B$

In presence of the detached flow zone at hub (operating point $\mathrm{A}$ ), the pressure gradients in the entry zone of the channel are small compared to the operating point $\mathrm{B}$ above the drop in the characteristic. Moreover the velocities are higher, leading to a strong flow passing the periodic interface in the lower part of the channel. 


\section{ANALYSIS OF RELATIVE STREAMWISE VORTICITY}

To visualize the secondary flow structures in the volume, a local definition of streamwise vorticity is used:

$$
\omega_{s}=\frac{\vec{\omega} \cdot \vec{c}}{c}=\frac{(\vec{\nabla} \times \vec{c}) \cdot \vec{c}}{c}
$$

To get only directional information about the angle between the vorticity vector and the velocity, the relative streamwise velocity is defined as follows:

$$
\omega_{s, r e l}=\frac{\vec{\omega} \cdot \vec{c}}{|\vec{\omega}||\vec{c}|}=\frac{(\vec{\nabla} \times \vec{c}) \cdot \vec{c}}{|\vec{\omega}||\vec{c}|}
$$

Plotting the relative streamwise vorticity for different discharge value in several planes that are rectangular to the main flow direction in the channel illustrates the development of the secondary flow structures.

a)

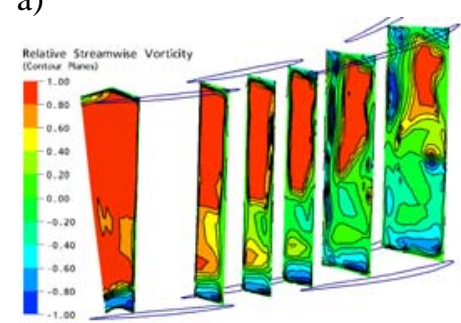

c)

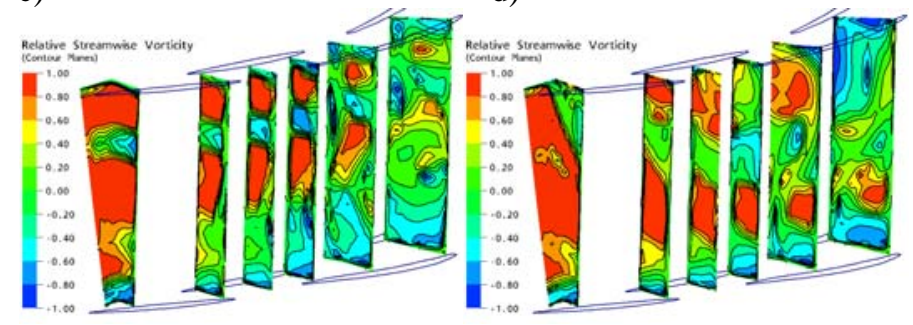

Fig. 15. Relative streamwise vorticity a) $\varphi=0.110$, b) $\varphi=0.105, c) \varphi=0.101, d) \varphi=0.100$

In this representation it is more obvious than on the skin friction line plots that the secondary flow structure near the hub is breaking up in two vortices with a counter rotating structure in between (c) before the drop in the characteristic curves and the appearance of the extended detached flow region at hub (d). The position where the structure breaks up corresponds to the minimum in the hydraulic energy profile that appears at $15 \%$ of the span near the hub at low discharge.

\section{CONCLUSIONS}

Numerical flow simulations with a stage interface model allow to reproduce a drop in the energy-discharge characteristic of a pump turbine in pump mode. The sudden change in the global performance parameters is related to a change of the secondary flow pattern in the diffuser channels. A symmetric flow configuration with two counter-rotating secondary vortices due to a minimum in rotor outlet hydraulic energy in the middle of the channel leads to local flow separation and does not affect the global performance. At lower discharge, a second minimum of pressure and kinetic energy at rotor outlet appears near the hub and gives rise to a second vortex structure in the channel. The related appearance of an extended low energy zone leads to a completely different flow direction through the guide and stay vane in which kinetic energy can not be recovered to pressure energy.

Regarding the validation in means of quantitative accuracy an unsteady simulation including the spiral casing has to be realized to verify the influence of the rotor-stator coupling approach and of the nonuniform circumferential distribution of pressure and discharge which has experimentally been observed in volute pumps at part load operation. As the observed phenomena are mainly triggered by the velocity and energy profiles at the rotor-stator interface, influence of the interface model must be further investigated as well as the impact of the turbulence models on the secondary flow in the rotor and the resulting profiles at the interface.

\section{ACKNOWLEDGMENTS}

This study is established within of the Project "Development of CFD procedures for improving the stability of head-capacity characteristics of turbo pumps" (CTI no 6806.1) whose participants are EPFL and Sulzer Pumps. The project is supported by the Federal Office for Professional Education and Technology (OPET). The authors wish to thank the staff of the Laboratory for Hydraulic Machines for providing support in the numerical work.

\section{REFERENCES}

[1] Ciocan, G. , 1998, "Contribution à l'analyse des écoulements 3D complexes en turbomachines" Phd Thesis, INPG, Grenoble,

[2] Guedes, A., Kueny, J.-L., Ciocan, G., Avellan, F., 2002, "Unsteady Rotor - Stator Analysis of Hydraulic Pump - Turbine : CFD and Experimental Approach". Proceedings of the 21st IAHR Symposium on Hydraulic Machinery and Systems, Lausanne, Switzerland, pp. 767-780, Lausanne

[3] Gugau, M., 2004, "Ein Beitrag zur Validierung der numerischen Berechnung von Kreiselpumpen", PhD Thesis, TU Darmstadt

[4] Ansys Canada Ltd, 2004, CFX-5 Solver Theory Turbulence and Wall Function Theory, Waterloo

[5] Tobak, M., Peake D. J., 1982, ”Topology of threedimensional separated flows” Ann. Rev. Fluid. Mech. 14, pp. 61-85

[6] Lakshminarayana, B., 1996, "Fluid dynamics and heat transfer of turbomachinery”, John Wiley and Sons, New York 